\title{
Oral delivery of gambogenic acid by functional polydopamine nanoparticles for targeted tumor therapy
}

Beilei Wanga,1, Tengteng Yuan ${ }^{\mathrm{a}, 1}$, Liqiong Zha ${ }^{\mathrm{a}}$, Yuanxu Liu ${ }^{\mathrm{a}}$, Weidong Chen ${ }^{\mathrm{a}}$, Caiyun

Zhanga, $^{\mathrm{a},}$, Youmei Bao ${ }^{\mathrm{b},{ }^{*}}$, Qiannian Dong a,*

aSchool of Pharmacy, Anhui University of Chinese Medicine, Anhui Academy of Chinese Medicine, Hefei 230012, Anhui, China

bSchool of Pharmaceutical Sciences, Southern Medical University, Guangzhou 510515, PR China

${ }^{1}$ These authors contributed to the work equally and should be regarded as co-first authors.

*Corresponding author. School of Pharmacy, Anhui University of Chinese Medicine, Anhui Academy of Chinese Medicine, Hefei 230012, Anhui, China

*Corresponding author. School of Pharmaceutical Sciences, Southern Medical University, Guangzhou 510515, PR China

E-mail: cyzhang6@ustc.edu.cn (C.Y.Zhang); youmei.bao@hotmail.com (Y.M. Bao); dongqn2005@126.com (Q.N. Dong) 


\section{NMR analysis and purity of Gambogenic acid (GNA)}

${ }^{13} \mathrm{C}$ NMR spectrum of GNA was recorded using a Bruker Advance $400 \mathrm{MHz}$ spectrometer with deuterated methanol (MeOD) as the solvent.

Purity of GNA was determined by HPLC (Agilent, Waldbronn, Germany) as Reference ${ }^{1}$. The column (ZORBAX Eclipse XDB-C8 column, $5 \mu \mathrm{m}$, $\phi 4.6 \mathrm{~mm} \times 150 \mathrm{~mm}$, Agilent, U.S.A.) was eluted with a binary mixture of acetonitrile and $0.20 \%$ acetic acid solution (volume ratio $85: 15$ ) at a flow rate of $1.0 \mathrm{ml} / \mathrm{min}$. Elution was monitored at $360 \mathrm{~nm}$ on a diode array detector. The injection volume was $10 \mu \mathrm{L}(5 \mu \mathrm{g} \cdot \mathrm{mL}-1$ gambogenic acid) and the column temperature was maintained at $25^{\circ} \mathrm{C}$.

\section{Fourier transform infrared spectra and differential scanning calorimetry}

Fourier transform infrared spectra (FTIR) was used to confirm the chemical reaction between PDA and FA. The samples were mixed with $\mathrm{KBr}$ powder. The infrared spectrometer of $400-4000 \mathrm{~cm}^{-1}$ wave number was utilized to scan the mixture by a Bruker Vector 22 FTIR spectrometer.

The thermograms of GNA and lyophilized GNA@PDA-FA SA NPs were investigated by means of differential scanning calorimetry (DSC) (Q200 F3 NETZSCH, Selb, Germany). About $5 \mathrm{mg}$ of sample was placed into an aluminium pan and sealed in the airproof conditions. The thermal behavior was performed from 30 to $200{ }^{\circ} \mathrm{C}$ at a scanning rate of $5^{\circ} \mathrm{C} / \mathrm{min}^{2}$.

\section{The water solubility of GNA and GNA@PDA-FA SA NPs}

Excess raw GNA was added to ultrapure water and centrifuged (12000 rpm for 10 min) after fully dissolving, then, the supernatant was injected in HPLC to get the water solubility of GNA.

\section{In vitro drug release of GNA@PDA-FA SA NPs in simulated gastrointestinal fluid}

In vitro drug release of GNA@PDA-FA NPs, GNA@PDA SA NPs and raw GNA were conducted with dialysis bags diffusion in release medium of simulated gastrointestinal fluid without enzyme. In detail, $1.75 \mathrm{~mL}$ GNA solution (0.7 mg GNA dissolved into $1.75 \mathrm{~mL}$ of ethanol),1.75 mL GNA@PDA NPs solution and $1.75 \mathrm{~mL}$ GNA@PDA-FA SA NPs solution (equivalent to 0.7 mg of GNA) were placed into three per-swelled dialysis bags ( $\leq 12 \mathrm{kDa}$, China). The bags were separately immersed in 100 
$\mathrm{mL}$ simulated gastric fluid for $2 \mathrm{~h}$ and then in simulated gastrointestinal fluid for another $7 \mathrm{~h}$ with stirring at $100 \mathrm{rpm}$ and $37 \pm 0.5{ }^{\circ} \mathrm{C}$, respectively. At predetermined intervals $(0.5,1,2,4,6,9,12,24,36,48,72 \mathrm{~h}), 500 \mu \mathrm{L}$ release aliquots medium was taken out for analysis, at the same time $500 \mu \mathrm{L}$ fresh release medium was added to the outer solution.

\section{Results}

\section{Detailed data on NMR analysis and purity of Gambogenic acid (GNA)}

Figure S1 showed the 13C NMR spectrum of GNA with the characteristic chemical shift at $\delta 16.1,17.6,17.9,20.6,21.0,22.0,25.2,25.6,(2 \mathrm{C}), 26.3,28.9,29.5$, 29.8, 39.7, 46.9, 48.9, 83.7, 84.0, 90.4, 100.6, 106.4, 107.4, 121.4, 122.0, 123.8, 128.1, 131.7, 133.6 (2C), 135.0, 138.1, 138.9, 155.9, 160.3, 163.5, 172.1, 179.1, 203.5, which were consistent with the literature data ${ }^{3}$.

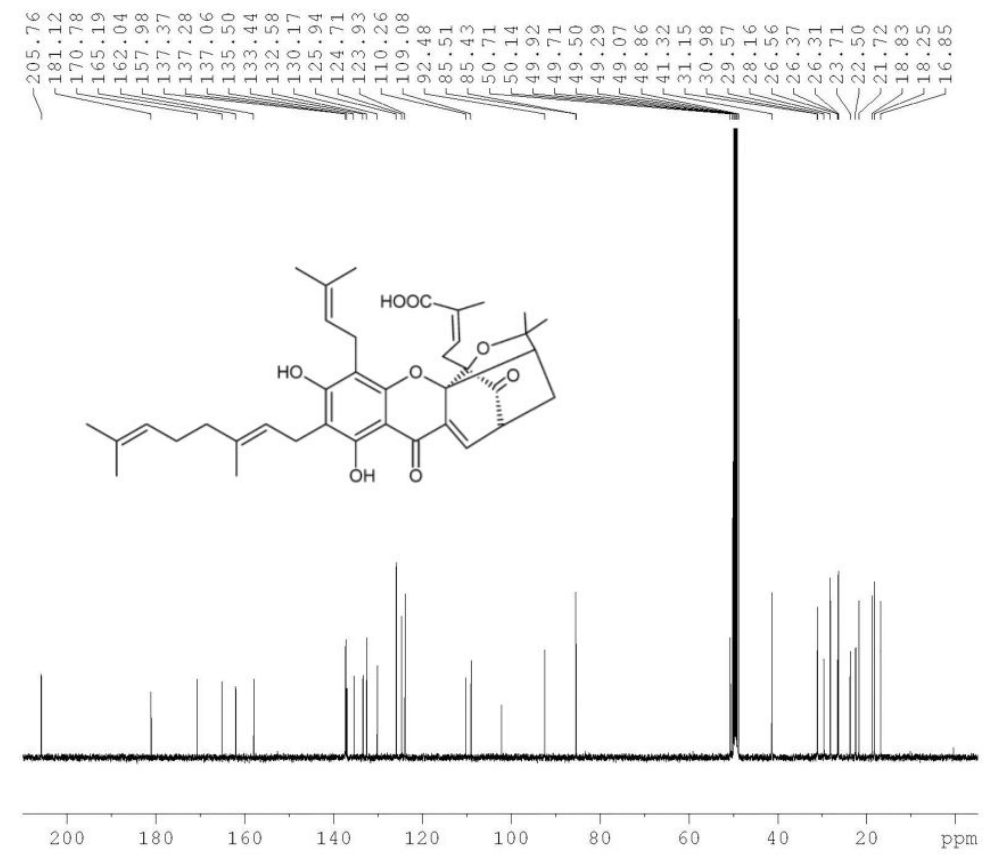

Fig S1. ${ }^{13} \mathrm{C}$ NMR spectrum of GNA

As shown in Fig S2, the purity of GNA was 98\% determined by HPLC. 


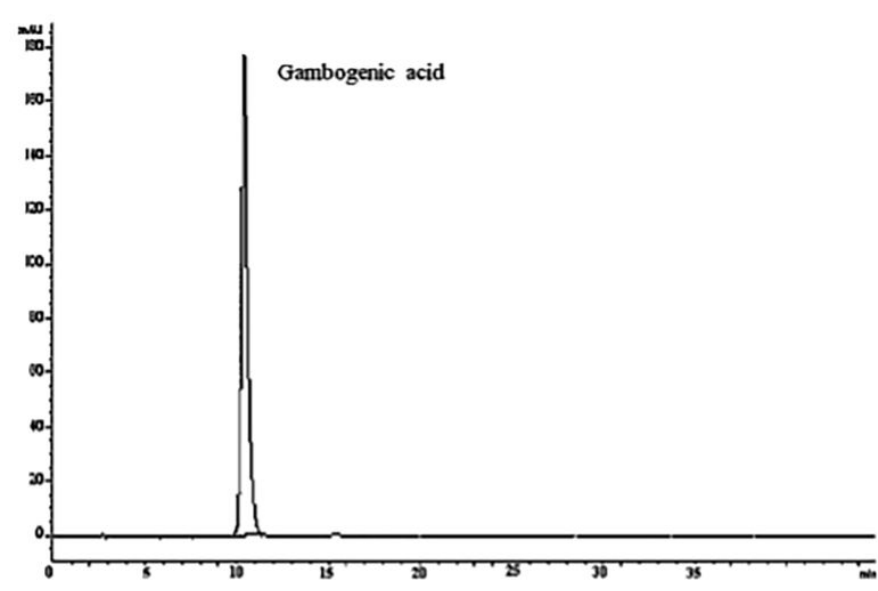

Fig S2. HPLC-UV Chromatograms of GNA

\section{Fourier transform infrared spectra and differential scanning calorimetry of GNA@PDA-FA SA NPs}

As shown in Fig S3(a), the FTIR of GNA exhibits strong absorption bands at 1450 $\mathrm{cm}^{-1}$ and $1630 \mathrm{~cm}^{-1}$ which are attributed to $\mathrm{C}-\mathrm{H}$ vibration of benzene ring and $\mathrm{C}=\mathrm{O}$ vibration of amide, respectively. The characteristic band at $2970 \mathrm{~cm}^{-1}$ was attributed to the carboxyl stretching vibration of GNA. FTIR in Fig S3(a) provided the information about the chemical composition of GNA@PDA NPs and GNA@PDA-FA NPs. The broad and strong absorption at $3400-3300 \mathrm{~cm}^{-1}$ was assigned to the stretching vibrations of $\mathrm{N}-\mathrm{H}$ and $\mathrm{O}-\mathrm{H}$. The absorption peak at $1651 \mathrm{~cm}^{-1}$ originated from $\mathrm{C}=\mathrm{O}$ of amide in stretching mode, and peaks at $1500 \mathrm{~cm}^{-1}, 1470 \mathrm{~cm}^{-1}$ and $1460 \mathrm{~cm}^{-1}$ were attributed to $\mathrm{C}-\mathrm{H}$ vibration of benzene ring ${ }^{4}$. The peak at $2943 \mathrm{~cm}^{-1}$ was assigned to $\mathrm{C}-\mathrm{H}$ vibration of alkane and became stronger after reaction with $\mathrm{FA}^{5}$. Compared to the FTIR of GNA, GNA@PDA NPs and GNA@PDA-FA NPs exhibited new absorption bands at $1020 \mathrm{~cm}^{-1}$ and $1090 \mathrm{~cm}^{-1}$, which are attributed to the stretching vibrations of C-N groups. Meanwhile, both absorption bands at $1020 \mathrm{~cm}^{-1}$ and $1090 \mathrm{~cm}^{-1}$ were significantly increased after FA function in GNA@PDA-FA NPs. These changes indicated that chemical reaction between FA and PDA may occur through the formation of amide.

DSC is a reliable method to provide insight into the possible interactions between PDA and FA. As shown in Fig. S3(b), a clear single melting endothermic peak of raw GNA was observed at $108^{\circ} \mathrm{C}$, which was in agreement with the reported data ${ }^{6}$. After 
GNA being captured in the PDA nanoparticles, the melting peak of GNA slightly shifted and turned broader in comparison with raw GNA. Moreover, a new endothermic peak appeared at $145{ }^{\circ} \mathrm{C}$ after the GNA@PDA-FA NPs formation, which indicated success conjugation of FA to the PDA core.
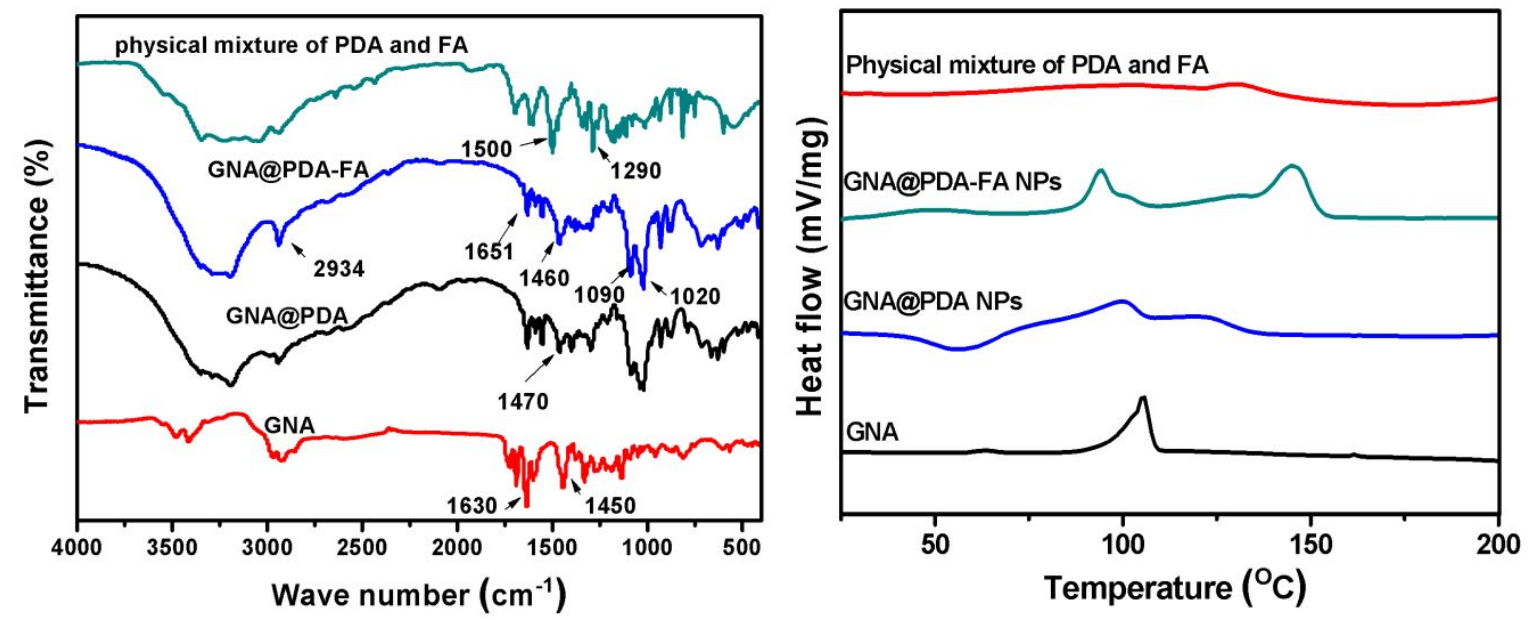

Fig S3. (a) FTIR spectra of physical mixture of PDA and FA, GNA@PDA-FA NPs, GNA@PDA NPs and raw GNA. (b) DSC of GNA, GNA@PDA NPs, GNA@PDA-FA NPs and physical mixture of PDA and FA, respectively.

\section{The water solubility of GNA and GNA@PDA-FA SA NPs}

Table S1 The water solubility of GNA and GNA@PDA-FA SA NPs $(n=3)$

\begin{tabular}{ccc}
\hline Samples & Pure GNA & GNA@PDA-FA SA \\
& & NPs \\
\hline Water solubility & \\
$(\mu \mathrm{g} / \mathrm{mL})$ & $0.27 \pm 0.05$ & $418.33 \pm 5.51$ \\
\hline
\end{tabular}

In this work, 0.4 mg GNA was loaded in 1 mL GNA@PDA-FA SA NPs solution (containing $0.4 \mathrm{mg}$ GMA, $0.2 \mathrm{mg}$ PDA, $0.2 \mathrm{mg}$ FA and $0.4 \mathrm{mg} \mathrm{SA}$ ) under optimal conditions, while the water solubility of pure GNA is about $0.27 \mu \mathrm{g} / \mathrm{mL}$ (Table S1). It can be seen that GNA@PDA-FA SA NPs greatly improved the water solubility of GNA. In vitro drug release of GNA@PDA-FA SA NPs in simulated gastrointestinal fluid

To investigate the stability and in vitro drug release of this oral formulation in gastrointestinal environment, the release of GNA@PDA-FA SA NPs were firstly carried out in simulated gastric fluid for $2 \mathrm{~h}$. The cumulative release percentages of 
GNA@PDA-FA SA NPs at $1 \mathrm{~h}$ and $2 \mathrm{~h}$ were $1.65 \%$ and $4.50 \%$, respectively. Then, we performed in vitro drug release of GNA@PDA-FA SA NPs in simulated intestinal fluid. As shown in Fig S4, accumulatively, up to $8.58 \%$ and $6.36 \%$ of GNA was released from GNA@PDA-FA NPs and GNA@PDA-FA SA NPs at 8h, respectively. The above results showed that GNA@PDA-FA NPs released a small amount of drug in simulated gastrointestinal fluid, indicating that NPs designed in this study can pass steadily through the gastrointestinal environment after oral administration.

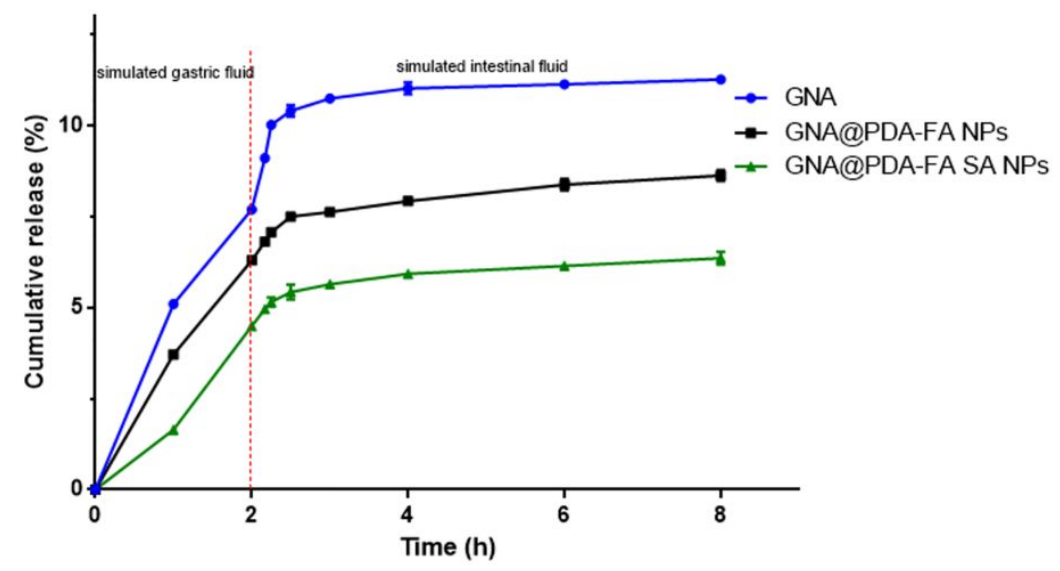

Fig S4. Release profiles of raw GNA, GNA@PDA-FA NPs and GNA@PDA-FA SA NPs in simulated intestinal fluid at $37 \pm 0.5^{\circ} \mathrm{C}(\mathrm{n}=3)$. 


\section{References}

1. Li, Q.; Cheng, H.; Zhu, G.; Yang, L.; Zhou, A.; Wang, X.; Fang, N.; Xia, L.; Su, J.; Wang, M.; Peng, D.; Xu, Q. Gambogenic acid inhibits proliferation of A549 cells through apoptosis-inducing and cell cycle arresting. Biological \& pharmaceutical bulletin 2010, 33, (3), 415-20.

2. Abdelraof, M.; Hasanin, M.; El-Saied, H. Ecofriendly green conversion of potato peel wastes to high productivity bacterial cellulose. Carbohydrate polymers 2019, 211, 75-83.

3. Asano, J.; Chiba, K.; Tada, M.; Yoshii, T. Cytotoxic xanthones from Garcinia hanburyi. Phytochemistry 1996, 41, (3), 815-20.

4. Bi, D.; Zhao, L.; Yu, R.; Li, H.; Guo, Y.; Wang, X.; Han, M. Surface modification of doxorubicin-loaded nanoparticles based on polydopamine with $\mathrm{pH}$-sensitive property for tumor targeting therapy. Drug delivery 2018, 25, (1), 564-575.

5. Yu, X.; Fan, H.; Wang, L.; Jin, Z. Formation of polydopamine nanofibers with the aid of folic acid. Angewandte Chemie (International ed. in English) 2014, 53, (46), 12600-4.

6. Liu, S.; Liu, J.; Cong, J.; Tong, L.; Zhang, Y.; Li, X.; Hou, J. Preparation of Neogambogic Acid Nanoliposomes and its Pharmacokinetics in Rats. Journal of the College of Physicians and Surgeons--Pakistan : JCPSP 2018, 28, (12), 937940. 